and calisthenics (20.4\%). Although the majority of patients visiting the ED for heat illness were discharged (91.9\%), 7.1\% were either hospitalized or transferred to higher care, with hospitalization being most common among males and patients aged more than 55 years.

Heat illness is a recognized threat to persons participating in sports and recreational activity. This analysis from the CDC provided epidemiologic information regarding heat illness that might be used for educational purposes, targeting persons 15 to 19 years old, more than 55 years old, males, coaches, and parents.

(Centers for Disease Control and Prevention. MMWR Morb Mortal Wkly Rep. 2011;60:977-980)

Prepared by Matthew Stewart, MD, University of Utah Emergency Medicine Resident, Salt Lake City, UT, USA.

\section{ARCHIVES OF SURGERY}

\section{Military Application of Tranexamic Acid in Trauma Emergency Resuscitation (MATTERs) Study}

This retrospective observational study assessed the effect of tranexamic acid (TXA) in combat injury on total blood product use, thromboembolic complications, and mortality. Tranexamic acid is a lysine analog that inhibits fibrinolysis and was shown to reduce mortality after civilian trauma in the CRASH-2 trial (Clinical Randomization of an Antifibrinolytic in Significant Hemorrhage). ${ }^{1}$ The MATTERs study reviewed 896 admissions due to combat injuries in Afghanistan that required at least 1 unit of packed red blood cells and was further subdivided into a massive transfusion cohort requiring 10 or more units of packed red blood cells within 24 hours. A total of 293 patients (32.7\%) received TXA within 1 hour of injury. Outcome measures were mortality, postoperative coagulopathy, and thromboembolic complications.

Administration of TXA had an absolute reduction for 30-day mortality of $6.5 \%(P<.3)$ in the overall cohort and $13.7 \%$ $(P<.004)$ in the massive transfusion cohort. Collectively, the TXA group had a higher injury severity score, greater transfusion requirements, and higher rates of thromboembolic complications; however, the authors noted that TXA was independently associated with survival (odds ratio 7.228) and less coagulopathy. Overall, the beneficial effect of TXA was much greater in patients with higher injury severity who were receiving massive transfusion. This study is limited by its retrospective design, differences of injury severity between groups, and difficulty following 30-day outcomes for Afghan natives. The authors conclude that TXA should be considered for combat injury patients with hemorrhage requiring extensive bloodproduct resuscitation to improve coagulopathy and overall survival.

(Arch Surg. 2011 [Epub ahead of print]) JJ Morrison, JJ Dubose, TE Rasmussen, MJ Midwinter.

Prepared by Matthew Stewart, MD, University of Utah Emergency Medicine Resident, Salt Lake City, UT, USA.

\section{Reference}

1. Roberts I. The CRASH-2 trial of an antifibrinolytic agent in traumatic haemorrhage: an international collaboration. Indian J Med Res. 2007;125:5-7.

\section{JOURNAL OF APPLIED PHYSIOLOGY}

\author{
Acute Mountain Sickness, Inflammation, and \\ Permeability: New Insights From a Blood Biomarker \\ Study
}

The authors of this article investigated the link between vasogenic edema associated with acute mountain sickness (AMS). One contributing factor to the development of vasogenic edema may be the disruption of the blood-brain barrier. Numerous mediators of inflammation and angiogenesis known to cause disruption of the blood-brain barrier can be modulated by hypoxia. This experimental study seeks to identify whether circulating biomarkers known to influence blood-brain barrier function are also related to AMS.

Twenty healthy volunteers (17 men and 3 women) were exposed to $4875 \mathrm{~m}$ in a hypobaric chamber. They participated in 3 separate trials, distinguished by pretreatment with placebo, acetazolamide, or dexamethasone using a randomized, doubleblinded, placebo-controlled crossover design. Venous blood was sampled at time points including 15 hours before, 0 hours, 0.5 hours, 4 hours, and 9 hours. Measurements included antiinflammatory biomarkers interleukin (IL)-1 receptor agonist (IL-1RA), IL-4, IL-10, heat shock protein (HSP)-70, adrenomedullin, proinflammatory biomarkers IL-6, IL-8, IL-2, IL$1 \beta$, substance $\mathrm{P}$, macrophage inflammatory protein- $1 \beta$, vascular endothelial growth factor, tumor necrosis factor-alpha, monocyte chemotactic protein-1, and serum matrix metalloproteinase-9. The AMS symptoms were evaluated using the Lake Louise Score (LLS).

Subjects with lower LLS had higher IL-1RA, HSP-70, and adrenomedullin compared with higher scoring AMS subjects. Acetazolamide raised IL-1RA and HSP-70 compared with placebo in higher scoring AMS subjects. Dexamethasone also increased HSP-70 and adrenomedullin in higher scoring AMS subjects. Macrophage inflammatory protein- $1 \beta$ was higher in higher scoring AMS subjects than lower scoring AMS subjects after 4 hours of hypoxia-dexamethasone minimized this difference. Other biomarkers did not appear to be related to AMS. Lower scoring AMS subjects demonstrated a marked antiinflammatory and antipermeability response that may have prevented downstream pathophysiological events leading to AMS. Conversely, higher scoring AMS subjects did not demonstrate an exaggerated inflammatory response.

(J Appl Physiol. 2011;111:392-399) CG Julian, AW Subudhi, MJ Wilson, et al.

Prepared by Derek Nusbaum, MD, Baylor College of Medicine Internal Medicine Resident, Houston, TX, USA. 